\title{
Designing epoxy viscosity for optimal mechanical performance of coated Glass Textile Reinforced Mortar (GTRM) composites
}

\author{
Cesare Signorini $^{\mathrm{a}, \mathrm{b}, *}$, Andrea Nobili $^{\mathrm{c}}$, Antonella Sola $^{\mathrm{c}}$, Massimo Messori $^{\mathrm{c}}$ \\ ${ }^{a}$ Dipartimento di Scienze e Metodi dell'Ingegneria, via Amendola 2, 42122 Reggio Emilia, \\ Italy \\ ${ }^{b}$ Dipartimento di Economia, Scienze e Diritto, Via Consiglio dei Sessanta 99, 47899 \\ Dogana, Republic of San Marino \\ ${ }^{c}$ Dipartimento di Ingegneria Enzo Ferrari, via Vivarelli 10, 41125 Modena, Italy
}

\begin{abstract}
Preliminary epoxy coating of the reinforcing fabric provides an effective approach for improving matrix-to-fabric strength in inorganic matrix composites. We investigate the effect of epoxy resin dilution in acetone on uni-axial tensile performance of coated alkali-resistant (AR) glass fabric embedded in a limebased matrix. Remarkably, it is found that dilution has a mixed effect on performance and this trend is consistently retrieved for strength, ductility and energy dissipation. Indeed, performance initially decays and then it suddenly raises to a level close to or even exceeding that of the undiluted specimens. It is postulated that this behaviour is caused by resin viscosity, that falls off exponentially with the dilution degree. Once a viscosity threshold is breached, epoxy is capable of penetrating inside the yarn and thereby prevents telescopic failure, that is the sliding of the outer over the inner glass filaments. Furthermore, the interphase surface area increases dramatically and this enhances performance and narrows scattering. Besides, optimal viscosity is reached at an unexpectedly high dilution degree, whence material cost is significantly reduced. A cost-toperformance comparison of common strengthening technologies is presented, which shows that diluted epoxy composites score comparably to FRPs. It is
\end{abstract}

\footnotetext{
${ }^{*}$ Corresponding author

Email address: cesare.signorini@unimore.it (Cesare Signorini)
} 
concluded that epoxy coating optimization plays an important role in designing inorganic matrix composites.

Keywords: TRM, Epoxy coating, dilution, optimization, viscosity

1

3

\section{Introduction}

Inorganic matrix composites, such as Textile Reinforced Mortar/Concrete (TRM/TRC), Fibre Reinforced Cementitious Matrix (FRCM) and Inorganic Matrix-Grid (IMG) are being extensively investigated as promising alternatives to the now traditional Fabric Reiforced Polymers (FRPs) [1]. Indeed, they present well-known advantages over FRPs, such as thermal stability [2], enhanced durability $[3,4]$, reversibility of intervention and affinity to traditional building materials $[5,6]$. Besides, from an environmental standpoint, the inorganic binder is certainly preferred over the organic matrix because it may be easily repaired, strengthened or, eventually, recycled $[7,8]$. On the other hand, inorganic binders offer weaker mechanical response and, therefore, they are unable to exploit the remarkable mechanical properties of the reinforcing fabric. As a result, failure is fragile and inconsistent, for it mainly occurs by internal delamination at the fabric-to-matrix interface [9]. On top of this, the inorganic binder is coarse and viscous and therefore it is unable to penetrate the small voids between the fabric filaments within the yarns. This leads to the possibility of telescopic failure, that occurs when the outer filaments (the so-called sleeve) adhere to the binder and yet they are capable of sliding over the inner filaments (the core). Although this failure mode is more ductile, for it is friction dominated, it still prevents the composite to reach its potential performance $[10,8]$.

Several techniques have been proposed to address the limits inherent to inorganic-based composites and, among these, fabric coating certainly appears as one of the most promising $[11,12,13,14]$. In particular, epoxy coating offers obvious advantages for it draws on the vast body of knowledge gained with FRP systems and, at the same time, minimizes the impact of the organic component. 
Remarkably, very few contributions are available in the literature concerning the actual role of epoxy resin at the fabric-to-matrix interface. Besides, for the most part, they report on the effect of the coating, with little investigation on the coating technique and formulation, nor on the action mechanisms. In [15], the effect of epoxy coating on tensile, pull-out and water absorption performance of TRC is presented. In [16], epoxy is applied at the lamination stage (wet coating) to create, together with sand, a thick intermediate layer between the carbon fabric and the inorganic matrix. In [10], two formulations for the epoxy coating are adopted which produce different coating thickness and this is found to strongly affect performance. In [2], epoxy coated TRM composites are exposed to high temperatures and mechanical performance appears little impaired until temperatures in excess of $200^{\circ} \mathrm{C}$ are reached. In general, the details of the mechanical action of the epoxy coating on the interphase strength remain unclear and likewise so for the optimal formulation and application strategies. Most importantly, we still need to understand how much we can benefit from epoxy coating in terms of mechanical performance and how well we fare in comparison with competing technologies. In this paper, following a simplest possible approach, we consider coating by a single epoxy resin with different degrees of dilution in acetone. The aim is to resolve the effect of interphase strengthening as opposed to yarn penetration. It is found that dilution weakens the interphase until interpenetration suddenly takes place and undiluted performance is restored or even surpassed. Finally, we present a simple cost effectiveness analysis according to which the optimal epoxy dilution places coated G-TRM alongside FRP in terms of cost-to-performance ratio.

\section{Materials and methods}

\subsection{Materials}

A commercially available premixed lime-based mortar (Kerakoll Spa, Geocalce Fino $\left.{ }^{\circledR}\right)$ is considered as the embedding medium for the reinforcing fabric; its nominal properties, as given by the manufacturer, are gathered in Table 1. 
Table 1: Mortar properties (Kerakoll Spa, Geocalce Fino ${ }^{\circledR}$ ), as given by the manufacturer

\begin{tabular}{lcc}
\hline Characteristic & Unit & Value \\
\hline Actual setting water content & $\%$ & $22 \div 23$ \\
Final density & $\mathrm{g} / \mathrm{cm}^{3}$ & 1.58 \\
Min. compression strength after 28 days & $\mathrm{MPa}$ & 15.0 \\
Min. flexural strength after 28 days (EN 196/1) & $\mathrm{MPa}$ & 5.0 \\
Min. support adhesion strength after 28 days & $\mathrm{MPa}$ & 1.0 \\
Aggregate maximum size & $\mathrm{mm}$ & 1.4 \\
Compression elastic modulus (EN 13412) & $\mathrm{GPa}$ & 9.0 \\
\hline
\end{tabular}

Table 2: AR-glass fabric properties.

\begin{tabular}{lcc}
\hline Characteristic & Unit & Value \\
\hline Yarn count & $\mathrm{tex}^{(*)}$ & 1200 \\
Net specific weight per unit fabric area & $\mathrm{g} / \mathrm{mm}^{2}$ & 300 \\
Fabric specific weight & $\mathrm{g} / \mathrm{cm}^{3}$ & 2.50 \\
Grid spacing (square grid) & $\mathrm{mm}$ & 12 \\
Equivalent thickness, $t_{f}$ & $\mathrm{~mm}$ & 0.06 \\
Ultimate strength along warp (with epoxy) & $\mathrm{MPa}$ & 1200 \\
Ultimate elongation along warp & $\mathrm{mstrain}^{(* *)}$ & 20 \\
Elastic modulus & $\mathrm{GPa}$ & 74 \\
\hline
\end{tabular}

(*) tex $=\mathrm{g} / \mathrm{km} ;{ }^{(* *)} 1 \mathrm{mstrain}=10^{-3} \mathrm{~mm} / \mathrm{mm}$.

56 Mortar characterization, through flexural and compression tests, is described in 57 Section 3.1.

58 A thermo-welded balanced open-square grid AR-glass woven fabric (Fibre ${ }_{59}$ Net SpA) is employed as the reinforcing phase. The zirconium oxide $\left(\mathrm{ZrO}_{2}\right)$

${ }_{60}$ sizing of the fibres ensures stability in the alkaline environment. The main ${ }_{61}$ geometrical and mechanical properties of this fabric, as declared by the manu-

${ }_{62}$ facturer, are reported in Table 2. We point out that, for better comparison, the same mortar and reinforcing fabric as in $[10,2]$ are considered.

\subsubsection{Fabric coating}

As detailed in Messori et al. [10], AR-glass fabric is preliminarily treated with a coupling agent, to enhance chemical compatibility with the epoxy coating. A $2 \%$ vol. aqueous solution of (3-Aminopropyl) triethoxysilane (99\%, Sigma-Aldrich) is prepared in a covered beaker and mixed on a magnetic stirrer for $15 \mathrm{~min}$. Cut-to-size fabric sheets are immersed in this silane solution 
Table 3: Epoxy coating compositions (normalized to $100 \mathrm{~g}$ of DER + acetone) and measurement of the actual polymer content $\left(w_{r}\right)$ with respect to the matrix $\left(w_{m}\right)$.

\begin{tabular}{c|cc|c|c|c}
\hline Dilution degree & D.E.R. $[\mathrm{g}]$ & Acetone $[\mathrm{g}]$ & DETA $[\mathrm{g}]$ & Resin $[\mathrm{g}]$ & $w_{r} / w_{m}[\%]$ \\
\hline $0 \%$ & 100 & 0 & 11.9 & 111.9 & 4.68 \\
$10 \%$ & 90 & 10 & 10.7 & 100.7 & 4.31 \\
$25 \%$ & 75 & 25 & 8.9 & 84.9 & 3.50 \\
$50 \%$ & 50 & 50 & 6.0 & 56.0 & 1.33 \\
$75 \%$ & 25 & 75 & 3.0 & 28.0 & 0.62 \\
$90 \%$ & 10 & 90 & 1.2 & 11.2 & 0.23 \\
\hline
\end{tabular}

for $1 \mathrm{~min}$ and then left to dry naturally at ambient temperature. Successively, the functionalized fabric is coated with epoxy resin obtained from high-purity bisphenol A diglycidylether (D.E.R. 332, DOW Chemicals). The aliphatic hardener diethylenetriamine (DETA 99\%, Alfa-Aesar) is adopted as curing agent. Alongside the undiluted epoxy, 6 dilution degrees are considered for the epoxy precursor and, to this purpose, technical acetone $\left(\left(\mathrm{CH}_{3}\right)_{2} \mathrm{CO}\right.$, Incofar Srl $)$ is adopted at 10, 25, 50, 75 and $90 \%$ weight ratios. To reduce the viscosity of the solution, D.E.R. is mixed with acetone on a magnetic stirrer at $50{ }^{\circ} \mathrm{C}$. The solution is subjected to ultrasonication for $5 \mathrm{~min}$ to promote homogenization. Finally, the curing agent is added and the solution is mixed for at least $15 \mathrm{~min}$ or until complete homogenization is reached. Successively, fabric sheets are immersed in the epoxy resin and than laid to harden for 7 days on a polypropylene support at ambient temperature. All the fabric sheets are weighted before and after coating in order to determine the polymer weight fraction in the finished composite, as listed in Table 3, which also summarizes the dilution degrees investigated in the present work. It is noteworthy that the total polymer weight does not exceed the threshold of $5 \%$ wt. defined by the most popular guidelines [17] and sharply decay around a dilution degree of $50 \%$, reaching a negligible value $(<1.5 \%)$. Besides, the polymer weight fraction does not correlate linearly with the dilution degree. Such evidence is in accordance with what found out in Section 4. 


\subsubsection{Specimen manufacturing}

Following Annex A of ICC AC 434 [18], 1-ply rectangular coupons are cast on an individual basis, to avoid cutting from a larger sheet. The manufacturing process takes place through the following stages (see $[4,6,13]$ for further details):

- Assembling of the dismountable formwork: the first set of equally-spaced 3-mm-thick constraining laths is placed onto a polyethylene support and silicone oil is applied;

- A first layer of natural hydraulic lime (NHL) mortar is laid in between the laths by manual wet lay-up and levelled on top with a scraper;

- Cut-to-size epoxy coated fabric sheets are laid and gently pressed onto the fresh mortar, Fig.1(a);

- The second set of 3-mm-thick constraining laths is attached on top of the first to provide guidance for the correct placing of the fabric as well as for a second layer of mortar;

- Mortar is laid in between the laths and levelled up, Fig.1(b).

Specimens are left moist-curing in a polypropylene bag for 7 days and then stripped from the formwork. Formwork disassembling facilitates the process of stripping. Successively, curing takes place in the laboratory environment for further 50 days. 7 days prior to mechanical testing, $100 \mathrm{~mm}$-long G-FRP tabs are glued at the specimen ends to accommodate the gripping system, Fig.1(c).

\section{Experimental investigation}

\subsection{Mortar characterization}

Three-point bending (3PB) tests are carried out on mortar prisms, according to the guidelines UNI EN 1015-07 [19], to precisely assess the mechanical performance of the lime-based mortar. Consequently, mortar specimens are cast in a $40 \times 40 \times 160 \mathrm{~mm}$ stainless steel mold and vibro-compacted. Specimen prisms are subjected to 7 day moist-curing and then stored to cure in a Memmert HP10 


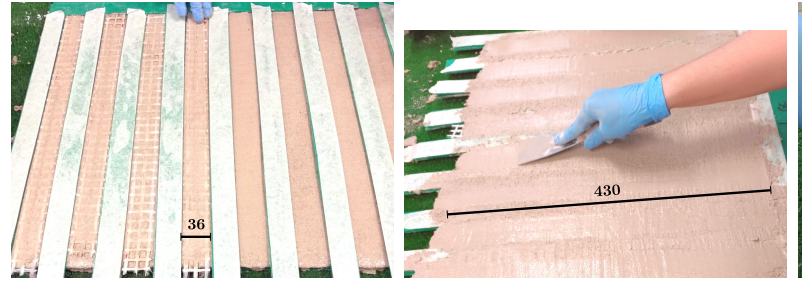

(a)

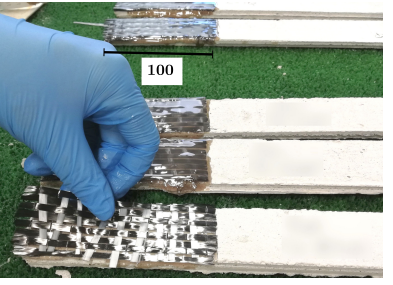

(c)

Figure 1: Specimen manufacturing protocol: (a) epoxy coated fabric sheets are placed onto the first layer of mortar, (b) application of the second layer of mortar, (c) C-FRP end tabs are glued to the specimens. Quotes are in $\mathrm{mm}$.

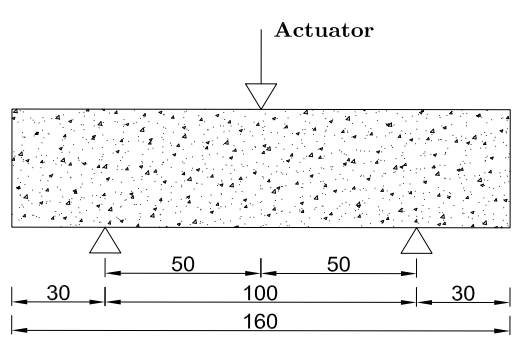

(a)

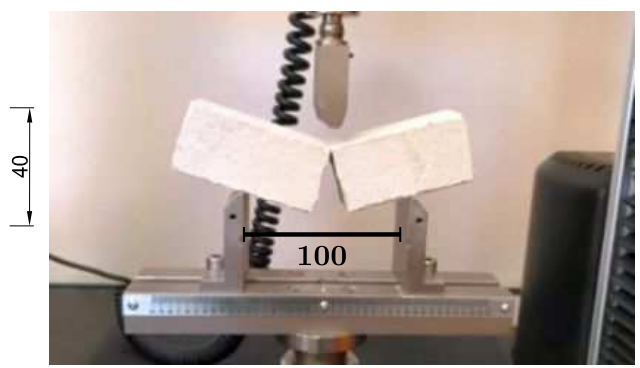

(b)

Figure 2: Three-point bending (3PB) test for mortar characterization: test geometry (a), test set-up and failed specimen (b). Quotes are in $\mathrm{mm}$. 


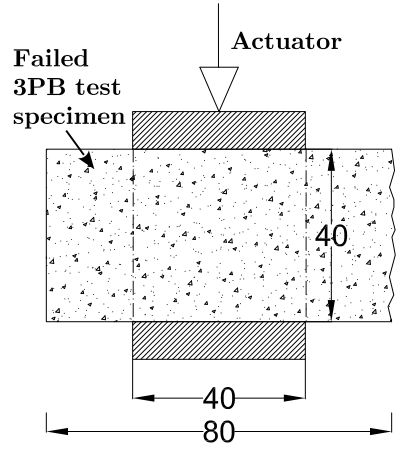

(a)

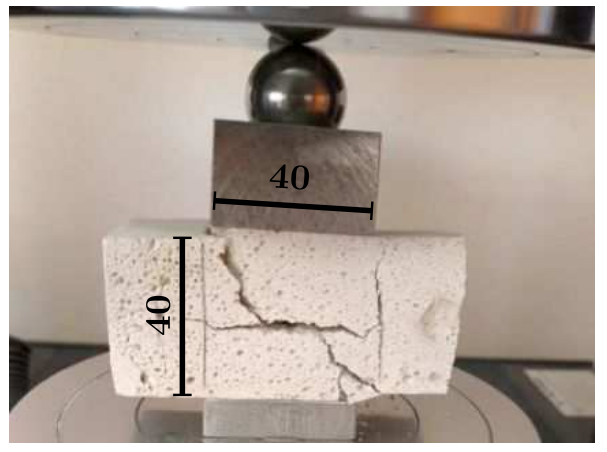

(b)

Figure 3: Compression test set-up (a) and failed specimen (b). Quotes are in $\mathrm{mm}$.

climatic chamber at $65 \%$ relative humidity $(\mathrm{RH})$ for further 21 days. Tests are performed through a Universal Testing Machine (UTM) at a nominal displacement rate of $1 \mathrm{~mm} / \mathrm{min}$ (equivalent to $50 \div 100 \mathrm{Ns}^{-1}$ ). The test geometry and set-up are shown in Fig.2.

Monotonic compression tests are performed on failed specimens after 3PB (Fig.3): either specimen end is compressed between two $40 \times 40 \mathrm{~mm}$ plates at a fixed displacement rate of $1 \mathrm{~mm} / \mathrm{min}$ (equivalent to $50 \div 500 \mathrm{Ns}^{-1}$ ). To rule out unwarranted bending effects which may arise owing to geometric irregularities in the specimen, a steel ball is placed right under the top steel plate of the UTM, see Fig.3. Flexural and compression ultimate strength and elastic moduli are obtained from the experimental curves. At least 6 specimens are tested both in compression and in flexure.

\subsection{Uni-axial tensile tests}

Uni-axial tensile tests are performed according to ICC AC434 [18] in a UTM equipped with a $30 \mathrm{kN}$ load cell. Wedge grips act on the end tabs and loading is applied by means of friction; wedges ensure adequate lateral pressure to avoid slippage between the specimen and the clamp (see [20] for a discussion on the effect of different clamping systems). A spherical hinge allows for self-alignment of the specimen and minimizes bending effects. Tests are conducted at a fixed strain rate of $2 \mathrm{mstrain} / \mathrm{min}\left(2 \cdot 10^{-3} \mathrm{~min}^{-1}\right)$, as prescribed in [21]. As in 


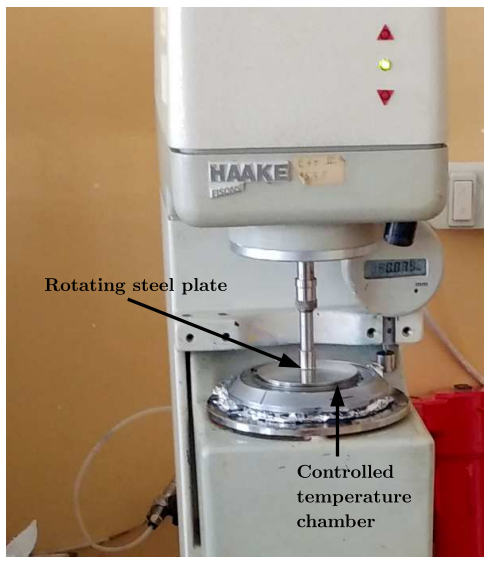

Figure 4: Rheometer test set-up

standard practice, stress and strain obtained from the test are referred to the fabric cross-section, $A_{f}$, and to the gauge length, $L_{g}=230 \mathrm{~mm}$,

$$
\sigma=\frac{P}{A_{f}}, \quad \varepsilon=\frac{\delta}{L_{g}} .
$$

40 Here, $P$ and $\delta$ are the load and the corresponding displacement, as measured

${ }_{141}$ by the UTM and corrected by DIC to eliminate wedge grip elongation (see also $[4])$.

\subsection{Digital Image Correlation data post-processing}

Tensile tests are monitored by Digital Image Correlation (DIC) with a 3 megapixel stereoscopic Dantec Dynamics optical system (Q-400). DIC allows to fine measure the actual displacement field of the specimen surface, that is previously sprayed with a fine black speckled pattern onto a white background.

\subsection{Optical and electron scanning microscopy}

Optical investigation at 35x magnification is performed in a Leica EZ4D stereo-microscope to qualitatively assess the coating distribution and its effect on interphase adhesion. A scanning electron microscope (SEM) Quanta-200 (Fei Company, The Netherlands) is also employed. 


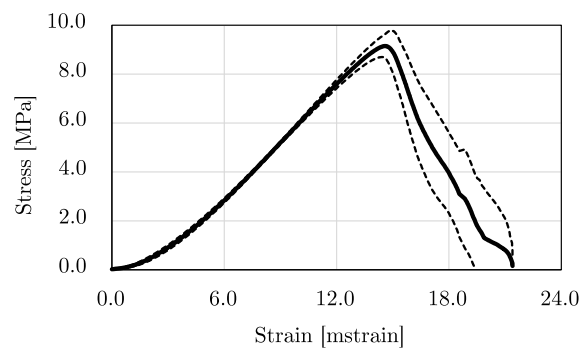

(a)

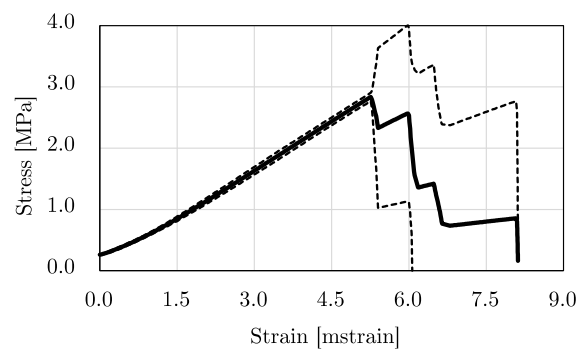

(b)

Figure 5: Mean stress-strain curve with \pm 1 standard deviation bands for compression (a) and bending (b) of lime mortar prisms. $\left(1\right.$ mstrain $\left.=10^{-3} \mathrm{~mm} / \mathrm{mm}\right)$

\subsection{Rheometer analysis}

Resin viscosity is measured, for different dilution degrees, through a HAAKE RS100 Rheostress rheometer (Fig.4), that applies to the fluid a nominal tangential stress, $\tau$, at constant temperature $(37 \pm 1)^{\circ} \mathrm{C}$. This temperature is chosen as to represent the actual conditions of fabric impregnation. The test program consists of an ascending ramp plus a descending ramp for the applied torque.

\section{Results}

\subsection{Mortar characterization}

The mean stress-strain curves for compression and bending of lime mortar prisms are reported in Figs.5(a) and (b), respectively. Data scattering is provided by \pm 1 standard deviation bands and it appears remarkably narrow for both compression and bending. Table 4 gathers the mean value of the mortar ultimate strength, $\mu(f)$, as well as of the secant modulus, $\mu(E)$, together with the relevant standard deviation, $\varsigma(\cdot)$, and coefficient of variation, $C V(\cdot)$. The characteristic value $(\cdot)_{k}$ is evaluated as for a normal distribution (with the socalled "2-sigma-rule", used in the construction of approximate $95 \%$ confidence intervals [22])

$$
(\cdot)_{k}=\mu(\cdot)-1.96 \varsigma(\cdot) .
$$

The elastic modulus is determined from the stress-strain curve as the slope of the secant line passing through $\left(\sigma_{0.6}, \varepsilon_{0.6}\right)$ and $\left(\sigma_{0.9}, \varepsilon_{0.9}\right)$, that are the stress- 


\begin{tabular}{|c|c|c|c|c|}
\hline $\begin{array}{l}\text { Characteristic } \\
\text { Unit }\end{array}$ & $\begin{array}{c}\boldsymbol{\mu}(\cdot) \\
{[\mathrm{MPa}]}\end{array}$ & $\begin{array}{c}\varsigma(\cdot) \\
{[\mathrm{MPa}]}\end{array}$ & $\begin{array}{c}\boldsymbol{C V}(\cdot) \\
{[\%]}\end{array}$ & $\begin{array}{c}(\cdot)_{k}(\mathrm{Eq} \cdot(2)) \\
{[\mathrm{MPa}]}\end{array}$ \\
\hline Compressive strength, $f_{c}$ & 9.2 & 0.5 & 5.4 & 8.2 \\
\hline Young Modulus, $E_{c, s}$ (Eq.(3)) & 802 & 41 & 5.1 & 723 \\
\hline Flexural strength, $f_{f}$ & 3.4 & 0.6 & 17.6 & 2.3 \\
\hline Flexural modulus, $E_{f, s}$ (Eq.(3)) & 539 & 13 & 2.4 & 512 \\
\hline
\end{tabular}

Table 4: Mechanical characterization of the lime mortar according to [19]. $\mu(f), \varsigma(f)$ and $(f)_{k}$ are the mean, the standard deviation and the characteristic value of the (assumed normally distributed) stochastic variable $f$ and they are connected through Eq.(2). $C V=\varsigma / \mu$ is the coefficient of variation

strain points at $60 \%$ and $90 \%$ of the ultimate strength [23]

$$
E=\frac{\sigma_{0.9}-\sigma_{0.6}}{\varepsilon_{0.9}-\varepsilon_{0.6}} .
$$

\subsection{Uni-axial tensile tests}

Fig.6 presents the mean stress-strain curve for each coating group alongside two reference curves expressing the mean performance in the uncoated and in the undiluted (alias 0\% diluted) epoxy group (the latter is taken from [10]). Remarkably, it appears that epoxy dilution in acetone little impairs the mean mechanical performance, which remains way superior to that of the uncoated group, particularly in terms of ductility. Further, most unexpectedly, performance is not a monotonic decreasing function of the dilution degree and, after an initial descending trend, it raises again and it reaches or even exceeds the undiluted performance.

To take a better insight into this unexpected behaviour, Fig. 7 presents the mean ultimate tensile strength (UTS) and strain for all groups alongside \pm 1 standard deviation bars. Looking at this figure, two interesting observations can be made:

1. the mean ultimate performance is a decreasing/increasing/decreasing function of epoxy dilution, which attains a local minimum at $10 \div 25 \%$-dilution and then raises up to a local maximum that occurs at $75 \%$ for strength and at $50 \%$ for strain; 

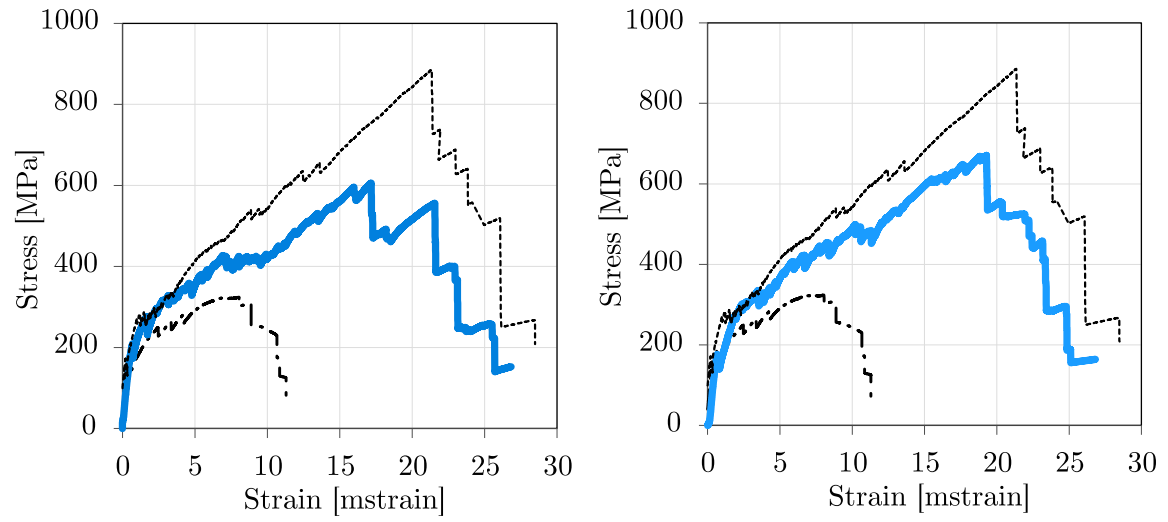

(a) $10 \%$

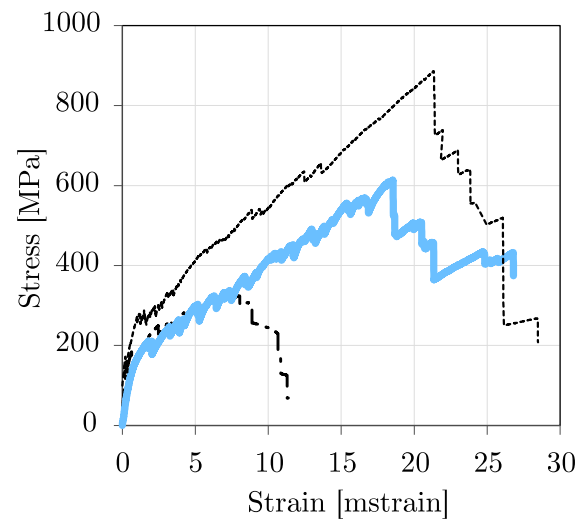

(b) $25 \%$

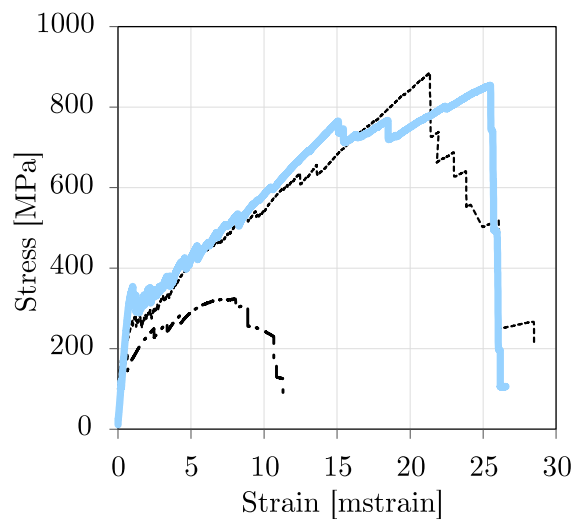

(c) $50 \%$

(d) $75 \%$

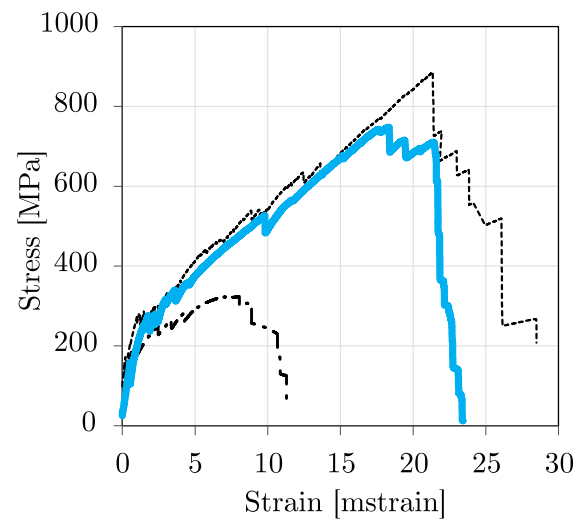

(e) $90 \%$

Figure 6: Mean stress-strain curves obtained in uni-axial tension of epoxy-coated G-TRM coupons for different dilution degrees (blue, solid lines), compared to the uncoated (black, dash-dotted lines) and to the undiluted (black, fine dashed lines) groups [24]. (1 mstrain = $\left.10^{-3} \mathrm{~mm} / \mathrm{mm}\right)$ 


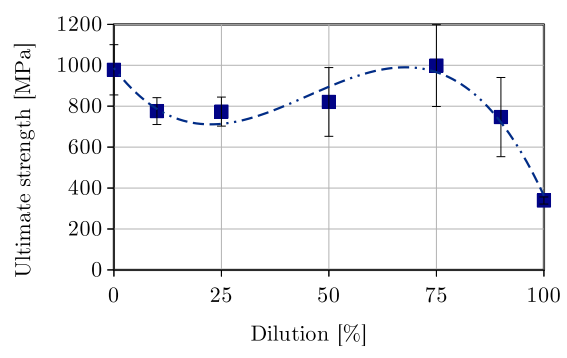

(a)

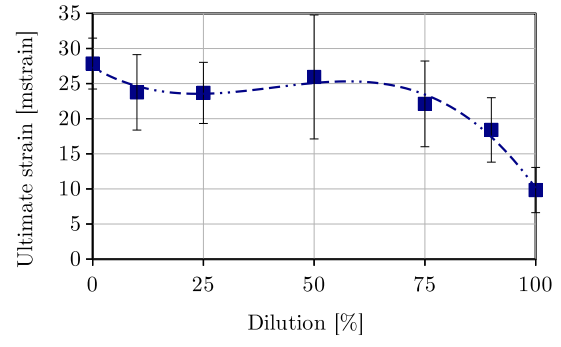

(b)

Figure 7: Mean ultimate strength (a) and strain (b) as a function of the dilution degree. \pm 1 standard deviation bars and cubic curve-fits are also plotted. $\left(1\right.$ mstrain $\left.=10^{-3} \mathrm{~mm} / \mathrm{mm}\right)$

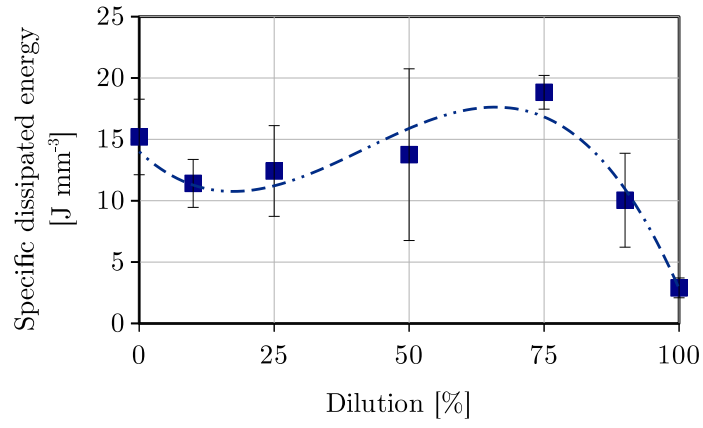

Figure 8: Mean energy dissipation per unit volume with \pm 1 standard deviation bars and cubic curve fitting 


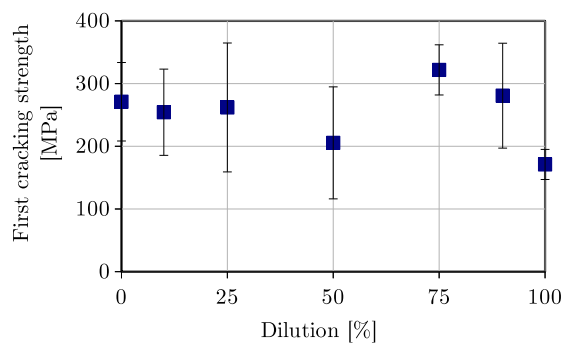

(a) FCS

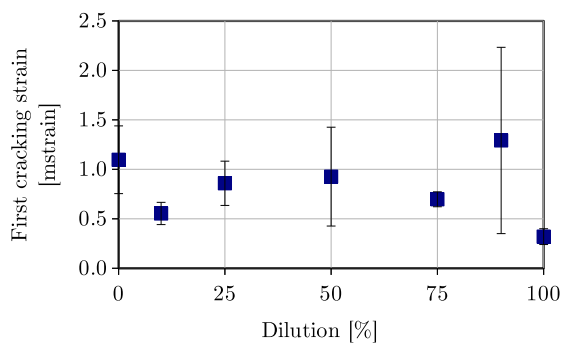

(b) Cracking strain

Figure 9: Mean first cracking strength (a) and strain (b) as a function of the dilution degree, with \pm 1 standard deviation bars. (1 mstrain $=10^{-3} \mathrm{~mm} / \mathrm{mm}$ )

2. strikingly, this maximum strength appears best performing (i.e. it is an absolute maximum), although it occurs in the presence of wider scattering when compared to the undiluted group;

3. data scattering is not monotonic increasing either and it is relatively stable across the groups.

It is important to emphasize that dilution affects strength and strain in a similar manner, which fact is a good indication that an underlying phenomenon is consistently being captured. Indeed, the same trend is confirmed by looking at the dissipated energy at failure, that is evaluated as the mean, within each group, of the area under the stress-strain curve. In this respect, Fig.8 is even more surprising, for best performance is again associated with $75 \%$ dilution and yet it now exceeds by more than $30 \%$ that of the undiluted group, while possessing remarkably narrow data scattering. This behaviour seems to suggest that a threshold dilution exists which triggers a beneficial effect on performance and scattering. This effect competes against and eventually overcomes the expected degradation of the epoxy coating associated with resin dilution.

Fig.9 plots the first cracking strength (FCS) and strain and once more it supports the assumption that dilution has a mixed bearing on mechanical performance. Here, however, the picture is less clear cut, owing to the uncertainty that is associated with the identification of the transition point. Indeed, tran- 


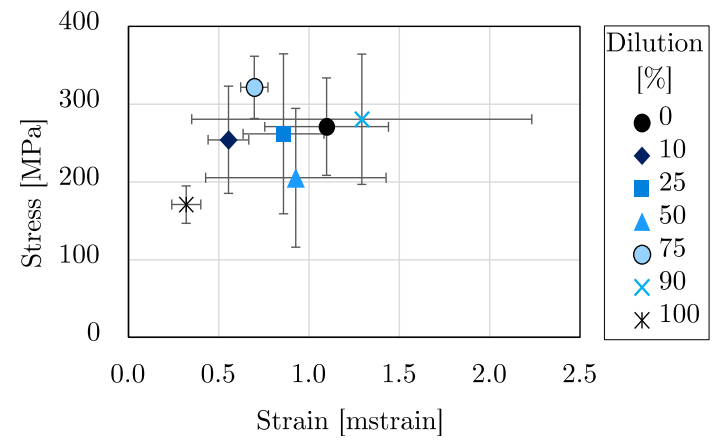

Figure 10: Transition points (TP) location as a function of the dilution degree with \pm 1 standard deviation bars. (1 mstrain $\left.=10^{-3} \mathrm{~mm} / \mathrm{mm}\right)$

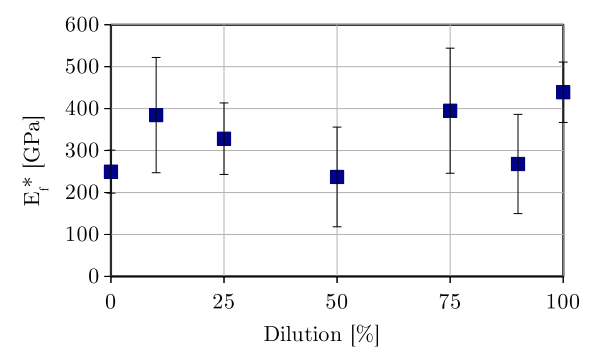

(a) Uncracked modulus

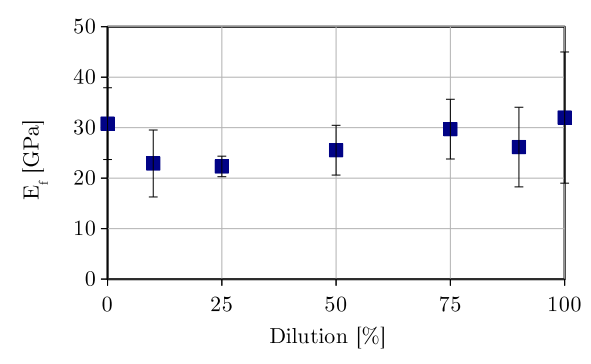

(b) Cracked modulus

Figure 11: Mean uncracked (a) and cracked (b) moduli as a function of the dilution degree with \pm 1 standard deviation bars 
sition point location is charted in Fig.(10) and it is interesting to observe that the highest stress and the least scattering again occurs at $75 \%$ dilution. Fig.11 shows the uncracked and the cracked elastic moduli, which reflects the matrix (see Tab.4) and the fabric moduli, respectively. As expected, little dependence on the dilution grade is demonstrated and yet the underlying trend can still be appreciated.

\subsection{Optical and Scanning Electron Microscopy}

Fig.12 presents the outcome of optical investigation of failed specimens at $35 x$. The effect of dilution emerges with great clarity in that individual fibres within the fabric yarn can only be appreciated at high dilution degrees, and to these fibres large mortar patches adhere. In contrast, at low dilution, fibres are deeply embedded in a sizeable lump of epoxy resin (that traps several air bubbles) to which only small mortar patches can adhere. Evidently, mortar adhesion occurs mainly at $50 \%$ and $75 \%$ dilution.

Along the same line and yet with greater detail, Fig.13 displays the outcome of SEM microscopy at 1000x. Indeed, at low dilution, scanty fibres emerge from the resin block which mainly surrounds the external filaments of the yarn, the so-called sleeve. To this block, scattered mortar patches adhere. In contrast, dilution reveals the glass fibres within the yarn, that the epoxy is eventually capable of penetrating. The coating thickness is substantially reduced to a thin layer to which mortar patches are diffusely attached. This adhesion is perhaps of poorer quality, yet it extends well inside the core of the fabric yarn and the surface area involved is greatly enlarged (Fig.14). Besides, telescopic failure, that is failure by sleeve filaments sliding over the core, is prevented. The dilution degree that triggers filaments penetration is the threshold level that significantly enhances mechanical response. Competing with this positive effect is the reduction of the epoxy content due to dilution, that weakens interphase adhesion and negatively affects performance. Of course, a mixed response is exhibited for intermediate dilution degrees. 


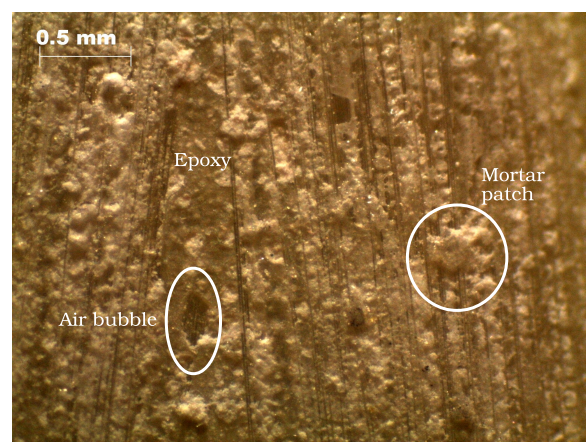

(a) $10 \%$

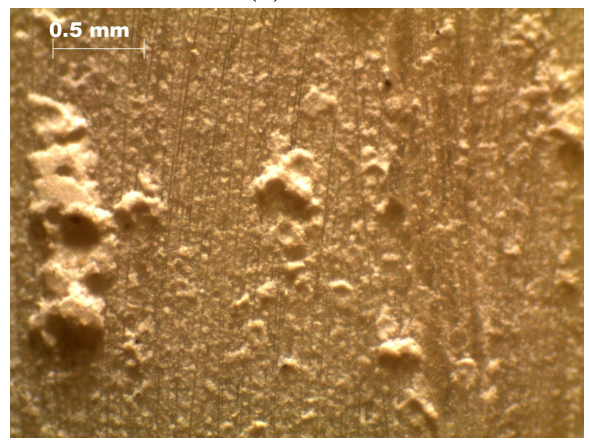

(c) $50 \%$

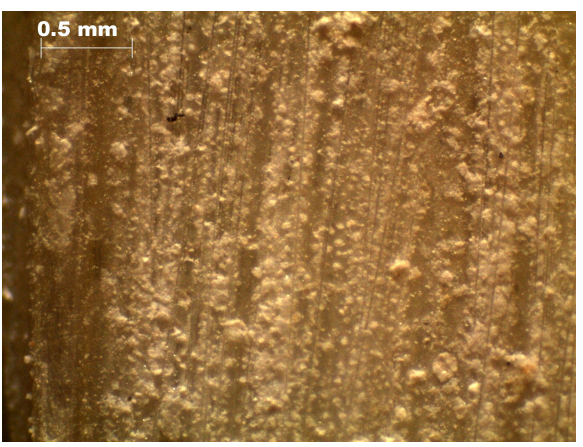

(b) $25 \%$

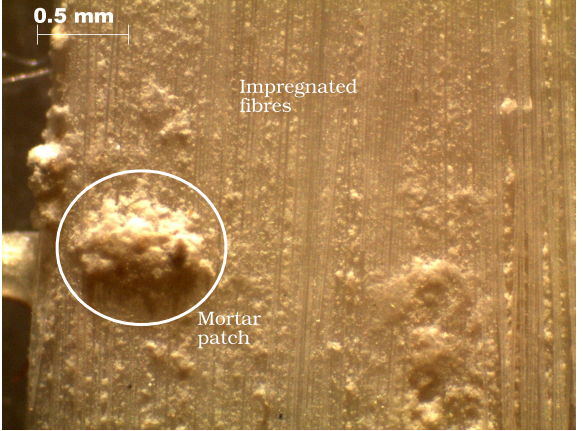

(d) $75 \%$

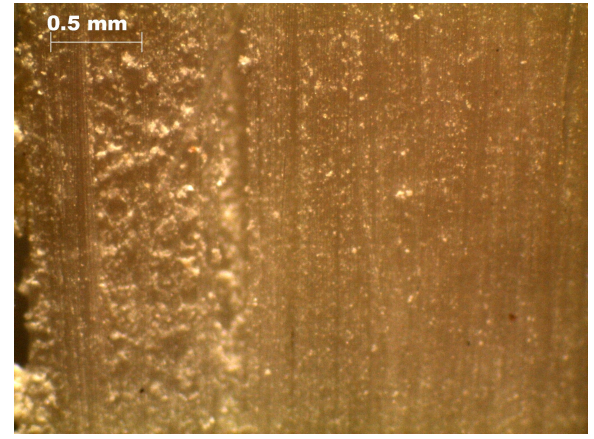

(e) $90 \%$

Figure 12: Optical microscopy of the fabric after failure at 35x magnification 


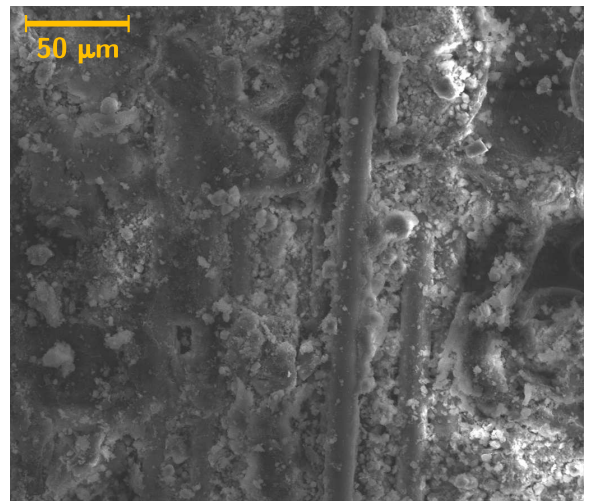

(a) $10 \%$

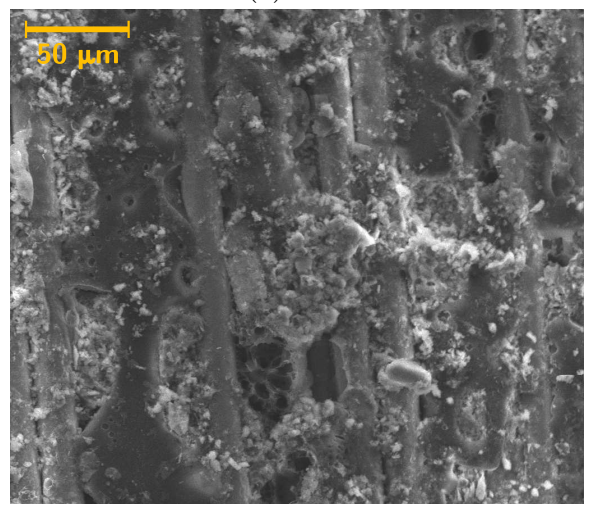

(c) $50 \%$

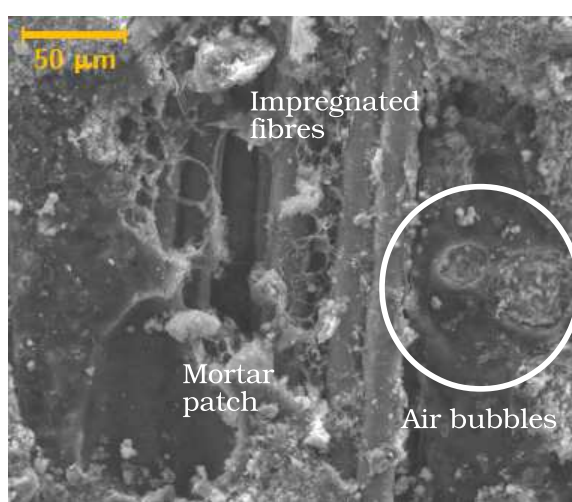

(b) $25 \%$

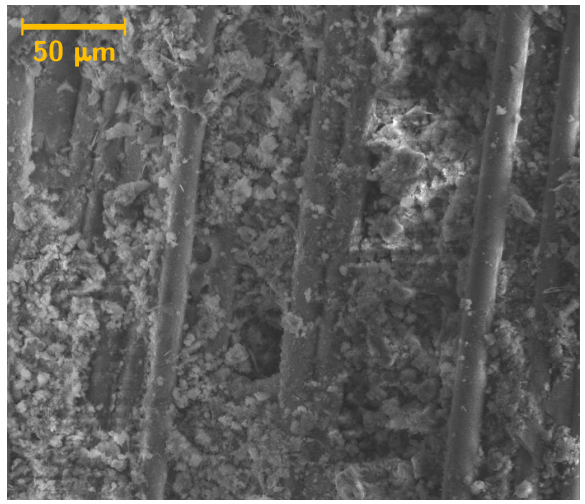

(d) $75 \%$

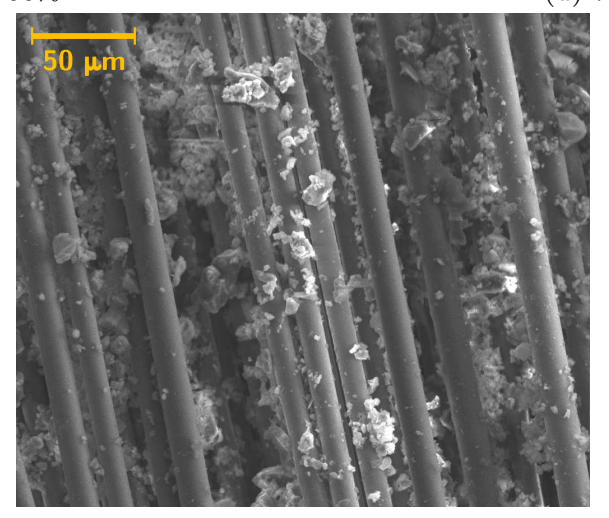

(e) $90 \%$

Figure 13: SEM of the fabric after failure at 1000x magnification 

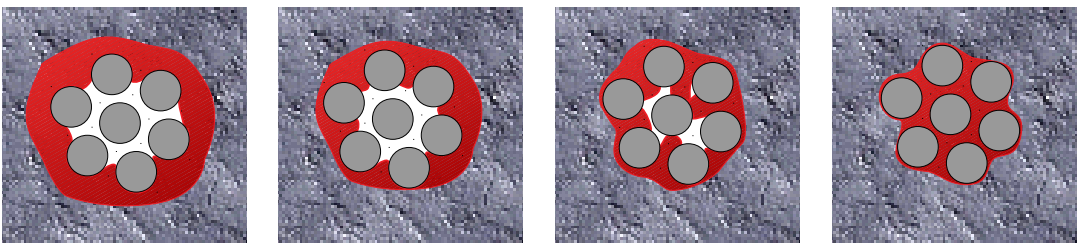

Figure 14: Penetration of the epoxy resin (red) in the voids between the filaments (gray) within the fabric yarn: dilution decreases viscosity and favours deep penetration

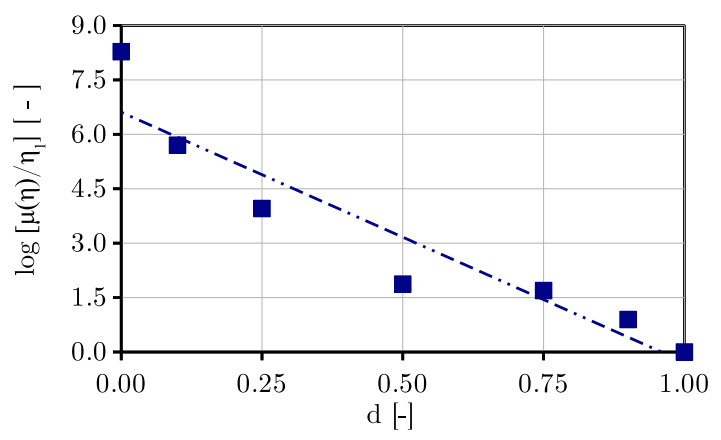

Figure 15: Viscosity measurement in logarithmic scale at $37^{\circ} \mathrm{C}$ as a function of the dilution degree. An exponential curve-fit is also proposed $\left(R^{2}=0.95\right)$

\subsection{Viscosity curve}

It is most striking that the beneficial effect of epoxy resin penetration in the yarn core occurs almost suddenly and at an unexpectedly large dilution degree. Indeed, the dilution threshold is located around $3: 1$ acetone to epoxy weight ratio. This outcome may be traced back to the exponential decay of epoxy viscosity as a function of acetone dilution $d$. Fig.15 plots the mean viscosity, $\mu(\eta)$, normalized to acetone viscosity $\eta_{1}$, superposed onto the exponential fit (logarithmic scale)

$$
\mu(\eta)=\eta_{1} \exp [-6.613(d-1)],
$$

where $0 \leq d \leq 1$ is the dilution degree. The viscosity at $d=1$ (i.e. the uncoated group) is let equal to acetone viscosity, namely $\eta_{1}=0.28 \mathrm{mPa} \cdot \mathrm{s}$ [25]. The fit shows good correlation $\left(R^{2}=0.9518\right)$. 


\begin{tabular}{c|ccc}
\hline Dilution & \multicolumn{3}{|c}{ probability of excedence [\%] } \\
100d [\%] & UTS & Strain & Energy \\
\hline 10 & 1.0 & 7.8 & 1.8 \\
25 & 0.4 & 9.8 & 8.4 \\
50 & 8.2 & 30.6 & 31.2 \\
75 & 53.6 & 46.2 & 89.2 \\
100 & 4.0 & 0 & 4.2 \\
\hline
\end{tabular}

Table 5: Permutation test results

\section{Resampling analysis}

We begin by considering the question whether the different behaviour that we see across the test groups is due to sampling or rather reflects some intrinsic variance. For this, we carry out a permutation test on the total count [26] and results are given in Tab.5. The permutation test works as follows: we compare two dilution groups, namely $0 \%$, that is the reference group, and $y \%$ and for this we bring them together in the set $A_{y}$. Then, we form $N=500$ permutations with repetition of 5 elements of $A_{y}$ and, for each of these, we compute the total count (sum of the elements) $s_{k}, k=1, \ldots, 500$. We count the number of permutations whose total count exceeds that of the reference group $0 \%$ and divide by $N$. We expect that, when difference is due to sampling, the total count of any permutation is located above/below the reference count $50 \%$ of the times. We see that this is never really the case for any group, although it may occasionally occur for a single characteristic. For instance, considering UTS and ultimate strain, we see that the reference group and $y=75 \%$ dilution are statistically equivalent, yet the latter still dissipates significantly more energy than the former. We conclude that results are statistically meaningful and an underlying process is being captured.

Next, we carry out a resampling analysis for each dilution group and consider the statistics of 5000 permutations with repetition of the original dataset. Fig.16 plot the mean UTS and ultimate elongation, normalized to the corresponding mean of the original sampling in the undiluted group, respectively $f_{0}$ and $\varepsilon_{0}$, 


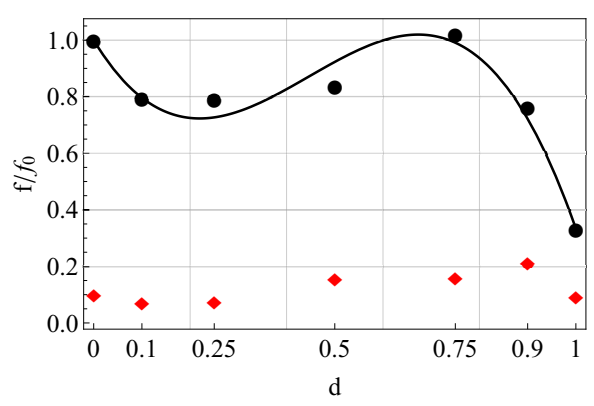

(a) UTS $f$

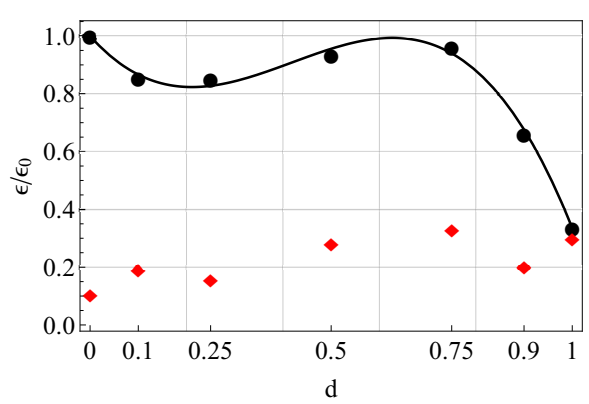

(b) ultimate elongation $\varepsilon$

Figure 16: Resampled mean tensile ultimate strength and elongation (black, circles) with mean CV (red, diamonds) as a function of the dilution degree $d$, normalized to the corresponding mean of the original sample for the undiluted group, respectively $f_{0}$ and $\varepsilon_{0}$. The cubic curve fit (5) is also shown

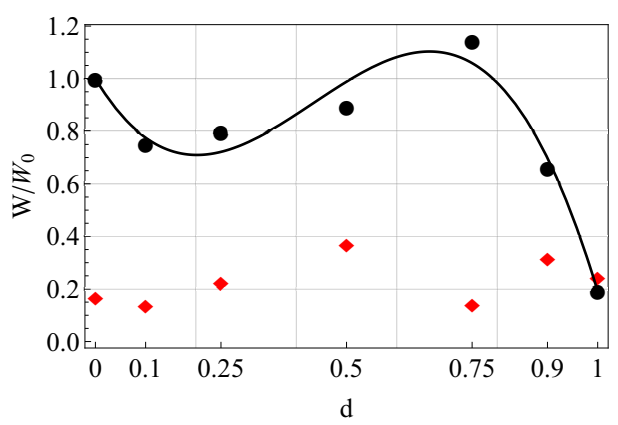

Figure 17: Resampled mean dissipated energy at failure $W$ (black, circles) and mean CV (red, diamonds) as a function of the dilution degree, normalized to the original sample mean dissipated energy of the undiluted group, $W_{0}$

superposed onto a cubic curve fit of the form (likewise for strain)

$$
\mu(f)=f_{0}\left[1+\left(f_{1}-1\right) x+a_{2} x(x-1)-a_{3} x\left(x^{2}-1\right)\right],
$$

where $f_{1}$ is the mean of the resampled uncoated group. A cubic curve-fit is chosen because $d \leq 1$ and any curve-fit turns into a polynomial in a Taylor sense. Remarkably, upon normalization, strength and elongation follow a very similar trend, whose resemblance supports the fact that a physical process is being portrayed. Besides, unless a very large dilution degree is adopted, performance rests above $80 \%$ of the uncoated group, before falling off steeply beyond $75 \%$ dilution.

Figs.17 presents the resampled dissipated energy at failure $W$, normalized 


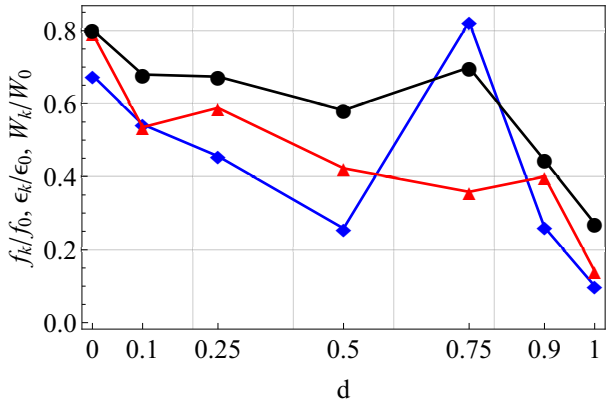

Figure 18: Resampled characteristic ultimate strength (black, circles), strain (red, triangles) and dissipated energy (blue, diamonds) as a function of the dilution degree, normalized to the corresponding mean values in the undiluted group

to the mean dissipated energy $W_{0}$ in the original undiluted sampling, and it shows that a $14 \%$ performance gain over the undiluted group is achieved at $75 \%$ dilution, that is accompanied by a very small CV. This behaviour, that seems to contrast that observed for strength and strain, may be explained noting that these are contravariant statistical variables, in the sense that when one increases, the other decreases and yet their "product" (i.e. the energy) remains the same.

With the resampling size $N=5000$, the population distribution is close to normality and we may safely consider characteristic values according to Eq.(2). Fig.18 presents the characteristic ultimate strength, strain and dissipated energy, normalized with respect to the corresponding mean value in the original sample of the undiluted group. We see that dilution has a significant negative effect on the characteristic ultimate strain, which drops in monotonic fashion. This is due to increasing standard deviation, see Fig.16(b). Conversely, strength and energy recover significantly at $75 \%$ dilution to the point that they behave almost as or better than the undiluted group. Such results support the idea that optimizing epoxy dilution and epoxy viscosity conveys remarkable benefit in terms of performance-to-cost efficiency.

\section{A simple cost-effectiveness analysis}

Fig.19(a) shows the total cost of raw materials per unit dissipated energy at failure. The material cost is a decreasing function of dilution, for acetone is 


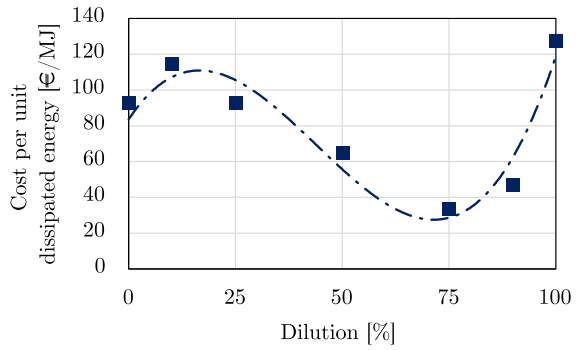

(a)

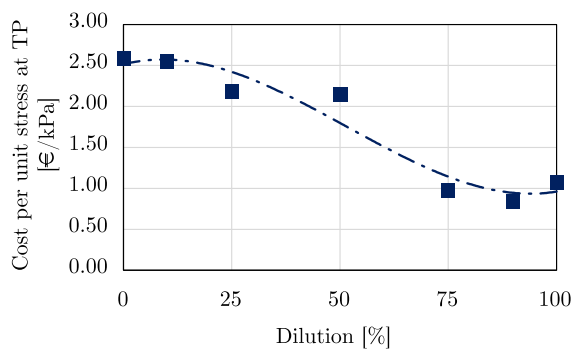

(b)

Figure 19: Mean cost per unit dissipated energy at failure (a) and per FCS (b) as a function of the dilution degree. A third-order polynomial curve-fit is also proposed.

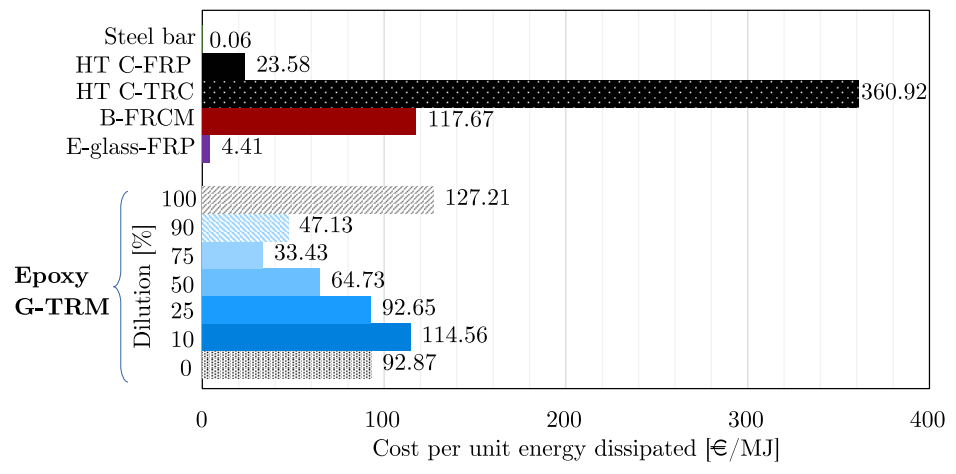

Figure 20: Comparison among strengthening and retrofitting technologies in terms of cost per unit dissipated energy (HT : high-tenacity, C : carbon, B : basalt, FRCM : fabric reinforced cementitious mortar)

far cheaper than epoxy, yet performance loss produces a competing trend. As expected, an absolute optimal point is met in correspondence of $75 \%$ dilution, where a $64 \%(74 \%)$ reduction in the cost-to-performance ratio is achieved, with respect to the undiluted (uncoated) group. Within this comparison, the undiluted group is only $37 \%$ better performing than the uncoated group, despite the vastly superior mechanical performance, see Fig.17. Unexpectedly, cost normalization in terms of FCS leads to a monotonic decreasing function, i.e. the cost reduction associated with dilution outweighs the performance decay in terms of FCS, see Fig.19(b). This result, among others, may justify the marginal diffusion of epoxy coating in the current TRM technology landscape.

Fig.20 presents an all-around cost-to-performance comparison among differ- 
ent strengthening and retrofitting technologies, in terms of mean material cost per unit dissipated energy. Costs for FRP and steel mesh are taken from 2018 data officially provided in [27]. FRP dissipated energy is obtained referring to the mean ultimate stress-strain values, as given by [28], and restricting the analysis to the linear regime. Mechanical response of steel is assumed to follow a bilinear model, with an initial elastic response until yield, followed by a linear hardening plastic phase until failure. Data for high-tenacity (HT) carbon (C) and basalt (B)-TRC are taken from Signorini and Nobili [29]. According to Fig.20, strengthening technologies may be ranked as follows. First, steel appears to be the best performing by far, manly in consideration of its marginal cost. This obvious result, which accounts for the large adoption of reinforced concrete, should be weighed against other design constraints, such as durability and feasibility. Then, FRP follows, in light of its low cost compared to the outstanding mechanical performance. Glass TRM comes third and we see that dilution is capable of dramatically reducing the cost-to-performance ratio to an extent that is comparable with high tenacity carbon FRP. In fact, $75 \%$ dilution produces an outstanding three-fold reduction in the cost-to-performance index compared to the undiluted group. Of course, different performance indices may be considered and costs greatly vary across countries, but the general idea that diluted epoxy coating may significantly increase the interest in TRM composites still holds. Basalt and carbon FRCM come last, although they score similarly to epoxy coated TRM. However, mechanical performance is generally little reliable in the absence of interphase strengthening [6].

\section{Conclusions}

We present the results of mechanical uni-axial traction tests on epoxy coated AR-glass Textile Reinforced Mortar (TRM) specimens. Spotlight is set on the role of the epoxy coating and its ability to penetrate the glass filaments within the yarn. To this aim, epoxy is diluted with acetone at 10, 25, 50, 75 and $90 \%$ weight ratios and performance is compared to that of undiluted and uncoated fabric. It is found that the effect of dilution is two-fold: on the one hand it de- 
creases the interphase strength and yet, on the other hand, it decreases viscosity exponentially and therefore enhances the epoxy penetration capability. These two effects are competing and, as a result, an optimal formulation is defined. Most remarkably, filament penetration occurs at a unexpectedly high dilution degree and it takes place through a rapid surge of mechanical performance. This burst is so intense that it compensates interphase weakening, to the extent that performance gets close to or even exceeds that of the undiluted specimens. This trend consistently emerges in the first cracking strength and strain, in the energy dissipation capability, in the cracked and uncracked moduli and in the turning point location alike. Besides, a simple cost-effectiveness analysis shows that dilution leads to a very substantial material cost reduction, which places this technology on an efficiency level comparable to that of FRPs. It is concluded that careful engineering of the epoxy coating can significantly contribute to the improvement of the overall performance of glass TRM composites.

\section{Acknowledgements}

Prof. Marcello Romagnoli is gratefully acknowledged for his contribution in carrying out the viscosity measurements.

\section{Funding}

This work was supported by the "Enzo Ferrari" Engineering Department (University of Modena and Reggio Emilia) through "FAR dipartimentali 2019"

\section{Declarations of interest}

None.

\section{Data availability}

The raw data required to reproduce these findings are available to download from http://dx.doi.org/10.17632/kbg7dz3yhx.1 


\section{References}

[1] V. Mechtcherine, Novel cement-based composites for the strengthening and repair of concrete structures, Construction and Building Materials 41 (2013) 365-373.

[2] M. Messori, A. Nobili, C. Signorini, A. Sola, Effect of high temperature exposure on epoxy-coated Glass Textile Reinforced Mortar (GTRM) composites, Construction and Building Materials 212 (2019) 765-774.

[3] R. Hempel, M. Butler, S. Hempel, H. Schorn, Durability of textile reinforced concrete, Special Publication 244 (2007) 87-108.

[4] A. Nobili, Durability assessment of impregnated glass fabric reinforced cementitious matrix (GFRCM) composites in the alkaline and saline environments, Construction and Building Materials 105 (2016) 465-471.

[5] U. Ebead, K. Shrestha, M. Afzal, A. El Refai, A. Nanni, Effectiveness of fabric-reinforced cementitious matrix in strengthening reinforced concrete beams, Journal of Composites for Construction 21 (2) (2016) 04016084.

[6] A. Nobili, F. Falope, Impregnated carbon fabric-reinforced cementitious matrix composite for rehabilitation of the Finale Emilia hospital roofs: case study, Journal of Composites for Construction 21 (4) (2017) 05017001.

[7] C. Signorini, A. Nobili, C. Siligardi, Sustainable mineral coating of alkaliresistant glass fibres in textile-reinforced mortar composites for structural purposes, Journal of Composite Materials (2019) 0021998319855765.

[8] B. Mobasher, Mechanics of fiber and textile reinforced cement composites, CRC press, 2011.

[9] F. Carozzi, C. Poggi, Mechanical properties and debonding strength of fabric reinforced cementitious matrix (FRCM) systems for masonry strengthening, Composites Part B: Engineering 70 (2015) 215-230. 
[10] M. Messori, A. Nobili, C. Signorini, A. Sola, Mechanical performance of epoxy coated AR-glass fabric Textile Reinforced Mortar: Influence of coating thickness and formulation, Composites Part B: Engineering 149 (2018) $135-143$.

[11] I. Van de Weyenberg, J. Ivens, A. De Coster, B. Kino, E. Baetens, I. Verpoest, Influence of processing and chemical treatment of flax fibres on their composites, Composites Science and Technology 63 (9) (2003) 1241-1246.

[12] C. Scheffler, S. Gao, R. Plonka, E. Mäder, S. Hempel, M. Butler, V. Mechtcherine, Interphase modification of alkali-resistant glass fibres and carbon fibres for Textile Reinforced Concrete II: Water adsorption and composite interphases, Composites Science and Technology 69 (7-8) (2009) 905-912.

[13] C. Signorini, A. Nobili, E. C. Gonzalez, C. Siligardi, Silica coating for interphase bond enhancement of carbon and AR-Glass Textile Reinforced Mortar (TRM), Composites Part B: Engineering 141 (2018) 191-202.

[14] C. Signorini, A. Sola, A. Nobili, C. Siligardi, Lime-cement textile reinforced mortar (TRM) with modified interphase, The Journal of Applied Biomaterials and Functional Materials 17 (1) (2019). doi:10.1177/2280800019827823.

[15] D. Dvorkin, A. Poursaee, A. Peled, W. Weiss, Influence of bundle coating on the tensile behavior, bonding, cracking and fluid transport of fabric cement-based composites, Cement and Concrete Composites 42 (2013) 919.

[16] J. Donnini, V. Corinaldesi, A. Nanni, Mechanical properties of frcm using carbon fabrics with different coating treatments, Composites Part B: Engineering 88 (2016) 220-228.

[17] ACI 549.4R-13, Guide to Design and Construction of Externally Bonded Fabric-Reinforced Cementitious Matrix (FRCM) Systems for Repair and 
Strengthening Concrete and Masonry Structures, American Concrete Institute, 2013.

[18] ICC-Evaluation Service, Acceptance criteria for masonry and concrete strengthening using fiber-reinforced cementitious matrix (FRCM) composite systems (AC434), Whittier, CA (2013).

[19] UNI EN 1015-11: Methods of test for mortar for masonry - Part 11: Determination of flexural and compressive strength of hardened mortar.

[20] J. Hartig, F. Jesse, K. Schicktanz, U. Häußler-Combe, Influence of experimental setups on the apparent uniaxial tensile load-bearing capacity of textile reinforced concrete specimens, Materials and Structures 45 (3) (2012) 433-446.

[21] RILEM Technical Committee 232-TDT, Test methods and design of textile reinforced concrete, Mater Struct 49 (12) (2016) 4923-4927.

[22] R. A. Fisher, Statistical methods for research workers, Oliver and Boyd, Edimburgh, 1925.

[23] D. Arboleda, Fabric reinforced cementitious matrix (FRCM) composites for infrastructure strengthening and rehabilitation: Characterization methods, Ph.D. thesis, University of Miami, open Access Dissertation. Paper 1282 (2014).

[24] C. Signorini, A. Nobili, A. Sola, M. Messori, Diluted epoxy-coatings for TRM composites: tensile tests raw data, http://dx.doi.org/10.17632/kbg7dz3yhx.3, , Mendeley Data, v3 (2019).

[25] G. Kaye, T. e. a. Laby, Tables of physical and chemical constants and some mathematical functions, Longmans, London, 1966.

[26] P. Good, Permutation tests: a practical guide to resampling methods for testing hypotheses, Springer Science \& Business Media, 2013. 
[27] Camera di Commercio di Modena, Building materials price datasheet 2018 (prezziario opere edili 2018), https://www.mo.camcom.it/tutela-delmercato/prezzi/prezzi-informativi-delle-opere-edili-in-modena (2019-0620).

[28] CNR-DT 200 R1, Guide for the design and construction of an externally bonded FRP system for strengthening existing structures, Italian National Research Council, Rome, 2013.

[29] C. Signorini, A. Nobili, Effect of aggressive environment exposure on mechanical performance of Steel-FRCM and Textile Reinforced Concrete (TRC), in: Esculapio (Ed.), ICCS21 - 21st International Conference on Composite Structures, 2018. 